BMJ Open

Ophthalmology

\section{Assessment of dysphotopsia in pseudophakic subjects with multifocal intraocular lenses}

To cite: Buckhurst $\mathrm{PJ}$, Naroo SA, Davies LN, et al. Assessment of dysphotopsia in pseudophakic subjects with multifocal intraocular lenses. BMJ Open Ophth 2017:1:e000064. doi:10.1136/bmjophth-2016000064

Received 27 December 2016 Revised 04 May 2017 Accepted 07 May 2017

\section{CrossMark}

${ }^{1}$ School of Health Professions, University of Plymouth, Plymouth, UK ${ }^{2}$ Ophthalmic Research Group, Life and Health Sciences, Aston University, Birmingham, UK ${ }^{3}$ Midland Eye, Ophthalmology, Solihull, UK

Correspondence to Professor James S Wolffsohn; j.s.w.wolffsohn@ aston.ac.uk

\section{ABSTRACT}

Aim To better understand the phenomenon of dysphotopsia in patients implanted with multifocal intraocular lenses (IOLs).

Methods Forty-five patients (aged $61.8 \pm 8.9$ years) implanted bilaterally with Tecnis ZM900 (diffractive multifocal), Lentis Mplus MF30 (segmented refractive multifocal) or Softec-1 (monofocal) IOLs (each $n=15$ ) 4-6 months previously and who had achieved a good surgical outcome were examined. Each reported their dysphotopsia symptoms subjectively, identified its form (EyeVisPod illustrations), quantified retinal straylight (C-Quant) and halo perception (Aston halometer). Retinal straylight and halometry was repeated by a second masked clinician to determine interobserver repeatability.

Results Subjective dysphotopsia ratings were able to differentiate Tecnis ZM900 from Lentis Mplus MF30 $(p<0.001)$, but not Lentis Mplus MF30 from groups implanted with Softec-1 $(p=0.290)$. Straylight was similar between the monofocal and multifocal IOL designs ( $\mathrm{p}=0.664)$. ZM900 IOLs demonstrated a uniform increase in dysphotopsia in comparison with the monofocal IOL $(p<0.001)$ as measured with the halometer, whereas sectorial refractive multifocal IOLS demonstrated a localised increase in dysphotopsia over the inferior visual field. Intraobserver repeatability was good for the straylight (intraclass correlation coefficients $($ ICC $)=0.77)$ and halometry $(\mathrm{ICC}=0.89$ ). There was no significant correlation between the subjective dysphotopsia severity and the straylight $(p=0.503)$ or halometry $(p>0.10)$ quantification or between straylight and the halo area $(p>0.30)$.

Conclusions Multifocal IOLs induce symptoms of dysphotopsia. Straylight did not differentiate between IOL designs, however halometry identified clear differences in light scatter due to the IOL optics. Whereas, subjective rating of overall dysphotopsia are not strongly associated with straylight or halo perception, the halometry polar diagram reflected the subjective descriptions of dysphotopsia.

\section{INTRODUCTION}

Understanding dysphotopsia is vital in achieving a high quality of life in all patients following multifocal intraocular lens (IOL) implantation. Dysphotopsia is a

\section{Key messages}

Dysphotopsia such as glare is commonly reported following multifocal intraocular lens implantation. but is generally only quantified subjectively as self-reported severity.

- The results of this study demonstrate that a clinically applicable halometry technique can quantify light scatter and differentiate between multifocal intraocular lens designs.

This new, rapid to perform, measure could be adopted in clinical practice to identify an individual patient's subjective tolerance to objective glare and to assess the amount of glare resulting from complex optical refractive correction.

disturbance of vision and includes light phenomena such as haloes, the subjective perception of a bright ring around a light source; it occurs due to optical non-conformities in the optical path such as cataract or optical boundaries. ${ }^{1}$ The current literature shows that implantation of a multifocal rather than a monofocal IOL can lead to unwanted optical phenomenon termed dysphotopsia. ${ }^{2}$ However, the literature comparing IOLs is equivocal as to which design features minimise dysphotopsia, due principally to the lack of objective methods for assessing dysphotopsia. The majority of studies examining dysphotopsia use various subjective questioning in the form of verbal interviews, ${ }^{3}{ }^{4}$ bespoke questionnaires, ${ }^{5}$ a validated questionnaire ${ }^{6}{ }^{7}$ or through subject-initiated complaints. ${ }^{8}$ An alternative method is to use graphics depicting visual demonstrations of different types of dysphotopsia allowing the subject to indicate which is most representative of what they perceive. ${ }^{9} 10$

Instruments designed to measure the effects of disability glare have also been used in multifocal IOL studies. Disability glare is usually quantified as the reduction in vision from a glare source present within 
the visual field and is due to the spread of light (or straylight) across the retina. ${ }^{11}$ The majority of techniques used to assess disability glare are composed of a central optotype chart of varying spatial frequency or contrast surrounded by a glare source. The intensity of the ambient light is changed or a glare source is added to determine the effect this has on measures of visual acuity or contrast sensitivity. Examples of this approach can be found in the form of the Brightness Acuity Tester (BAT; Marco, Florida, USA), Mesoptometer II (Oculus Optikgera"te GmbH, Wetzlar-Dutenhofen, Germany) or digital view-in visual testing units, such as the Optec 6500 (Stereo Optical Co, Chicago, Illinois). Several custom-built glare testing units have also been developed. ${ }^{12}{ }^{13}$ However, these testing units do not quantify the extent of dysphotopsia and the literature shows variable results. Similar studies involving the $C$-Quant (Oculus Optikgera"te GmbH, Wetzlar-Dutenhofen, Germany), an instrument for evaluating the quantity of ocular straylight, have shown similar variability with a marked difference in straylight identified in some studies, ${ }^{14-16}$ but not others, ${ }^{2}{ }^{17-19}$ and higher straylight with diffractive designs than refractive and segmented designs. ${ }^{20}$ The difference in light scatter between a monofocal IOL and diffractive bifocal and trifocal IOLs has been recently reported with the light-distortion analyser (HLMP-CW47-RU000, Agilent Technologies) an experimental device consisting of a central white light-emitting diode (LED) surrounded by 240 small, white LEDs distributed in 24 meridians 15 degrees apart; ${ }^{21-23}$ a difference was found between them, but this was not correlated with visual acuity. The disparity between reported dysphotopsia and the results recorded with glare testing units may be due to the optical properties of multifocal IOLs. Pupil size does not seem to affect straylight measures, but this has only been assessed in spherical IOLs. ${ }^{24}$ Dysphotopsia due to multifocal IOLs may primarily be the result of a second out of focus image being present on the retina rather than diffuse straylight over the retinal surface (scatter affecting a much broader area) as induced by conditions such as cataract. ${ }^{13}{ }^{17}$ To measure the qualitatively described light surrounding retinal blur circle or halo, several instruments often referred to as 'halometers' have been created. ${ }^{25-27}$ These devices measure the size of a photopic scotoma created by a central glare source.

The purpose of this study was to examine the phenomenon of dysphotopsia in patients implanted with two multifocal IOL designs (refractive and diffractive) and to compare subjective symptoms to the quantification of straylight and halo size. A standardised preoperative measure of halo size compared with subjectively reported symptoms due to cataract, might help to identify individuals who are more likely to better tolerate dysphotopsia effects potentially induced by multifocal IOLs.

\section{METHODS}

Patients were recruited from Solihull Hospital and Midland Eye (Solihull UK). The NHS local research ethics committee of Solihull approved the study, and informed consent was obtained from each patient. The consequences and details of the study were explained to each patient, and the research followed the tenets of the declaration of Helsinki.

The recruitment inclusion criteria were patients requiring bilateral cataract surgery, with an expected postoperative best corrected distance visual acuity of at least $0.1 \mathrm{log}$ of the minimum angle of resolution (logMAR), not having any ocular pathology or previous surgery, with corneal astigmatism less than $1.00 \mathrm{D}$, aged between 40 and 70 years, deemed suitable by the treating surgeon and willing to be implanted with multifocal IOLs. The IOLs, implanted bilaterally, depended on the availability of the IOLs: Tecnis ZM900, addition power $+4.0 \mathrm{D}$ at the IOL plane (Abbott Medical Optics, Santa Ana, California); Lentis Mplus, addition power +3.0D (MF30) at the IOL plane (Oculentis, Berlin, Germany); and Softec-1 monofocal IOL (Lenstec, St Petersburg, Florida).

Preoperatively, biometry was conducted with an IOLMaster (Carl Zeiss Meditec AG, Jena, Germany) running V.5.2 analysis software and a NIDEK $O P D$ Scan II wavefront aberrometer (Optical Path Difference Scanning System II; NIDEK Co, Gamagori, Japan) to measure axial length and corneal power. To determine IOL power, the Hoffer Q IOL formula was used for short axial lengths $(<22 \mathrm{~mm})$ and the SRK/T was used for all other axial lengths; emmetropia was the target in all cases (College of Ophthalmologists guidelines, 2010).

All operations were performed by one of three surgeons (SS, TAK and MTB) experienced in fitting multifocal IOL, under topical or local anaesthetic. A $2.85 \mathrm{~mm}$ clear corneal incision, widening to $3.20 \mathrm{~mm}$ after injection, was placed on the steepest corneal axis by all surgeons to reduce residual levels of postoperative astigmatism. Phacoemulsification, aspiration and irrigation were performed through a $5.50 \mathrm{~mm}$ capsularhexis using the Millennium Phacoemulsification System (Bausch and Lomb, Rochester, New York, USA.). The surgeons used personalised A constants based on their documented surgically inducted refractive error for each lens type and used the same type of corneal incisions. The number of cases performed in each type of IOL was balanced for each of the three surgeons. All IOLs were implanted into the capsular bag.

Patients were re-examined 4-6 months after surgery with slit-lamp biomicroscopy. All patients were asked to evaluate their experience of photopic phenomenon post-IOL implantation using the question 'Please can you rate your experience of glare or unusual phenomenon around lights such as haloes on a scale of 0 to 10; zero indicating no glare experiences and 10 denoting extreme symptoms'. 


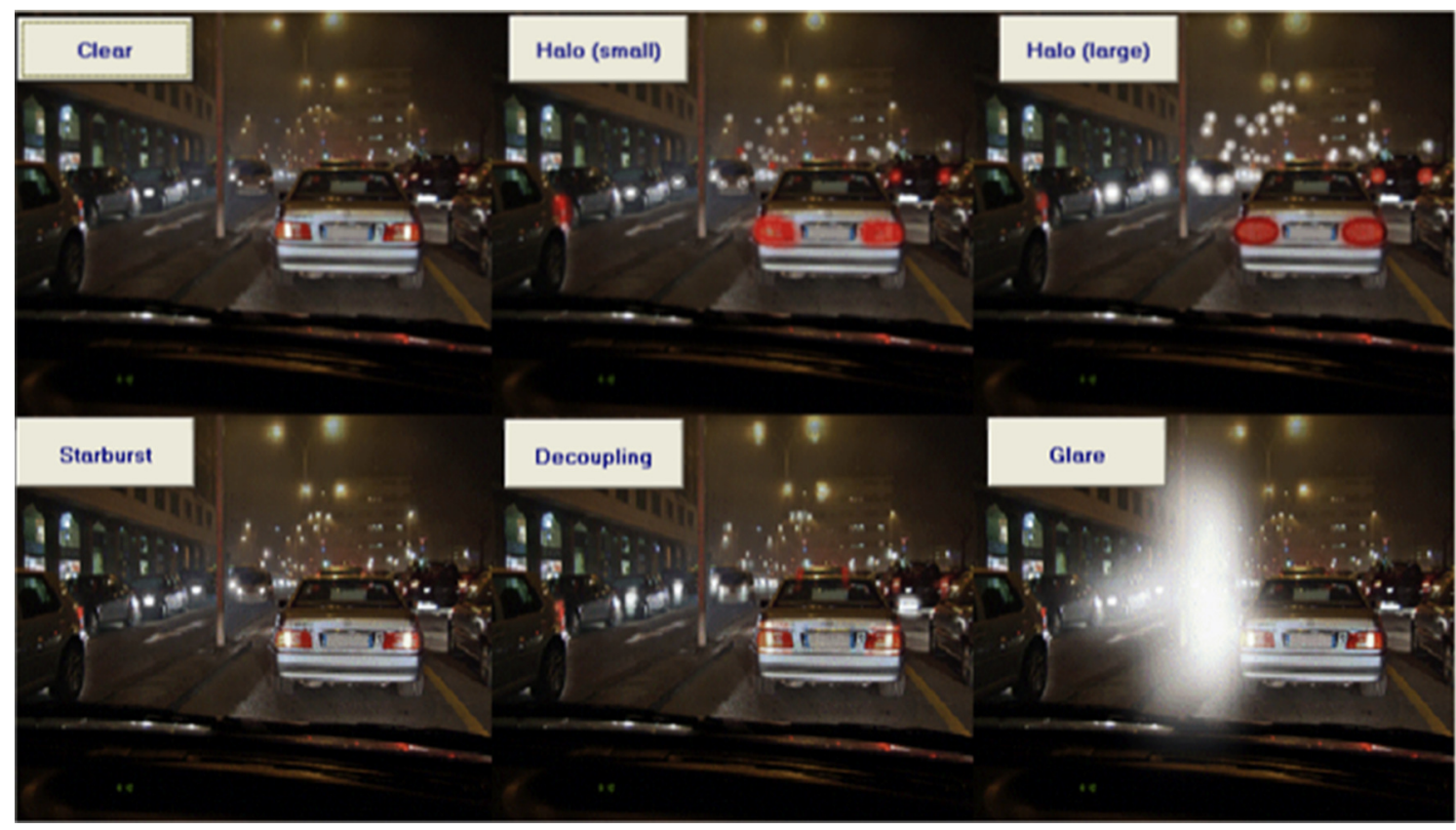

Figure 1 The EyeVisPod (PGB, Milan, Italy) graphical illustration depicting dysphotopsia (with the kind permission of EyeVisPod).

Each subject was shown a set of dysphotopsia illustrations as depicted on the EyeVisPod programme (PGB, Milan, Italy). Subjects were instructed to indicate the illustration which best represented their experience of dysphotopsia (figure 1).

Retinal straylight was measured three times in each eye on each patient with the $C$-Quant using its compensation comparison method (a flickering ring induces straylight at the fixation point in its centre, and this is compared with a comparison field). ${ }^{28}$ The average of three readings was taken as the measurement of straylight.

Halometry was conducted both monocularly (right eye) and binocularly with the Aston halometer. ${ }^{27}$ The halometer comprises a bright LED in the centre of an iPad V.4 (Apple, Cupertino, California) at $2 \mathrm{~m}$ working distance in a dark room. Letters subtending $0.21^{\circ}(500$ Weber contrast units) are moved away from the central glare source in $0.05^{\circ}$ steps in each of eight directions of orientation separated by $45^{\circ}$. The first eccentricity at which the letter could be correctly identified twice was recorded, with the letter randomised between presentations.

Retinal straylight and Aston halometry was repeated at the same visit by a second masked clinician to determine interobserver repeatability.

\section{Data analysis}

Sample size calculation to achieve significance below $\mathrm{p}=0.05$ with $80 \%$ power and using the halometer variability of $0.01^{\circ}$ to detect a $0.01{ }^{\circ}$ difference and CQuant of $0.14 \log (\mathrm{s})$ to detect a $0.15 \log (\mathrm{s})$ difference ${ }^{27}$ identified a minimum of 12 patients were required in each group (Statistical Solutions LLC). Patients without a good visual outcome including posterior capsular opacification, rhexis phimosis or marked capsule fibrosis as observed using a slit-lamp biomicroscope were excluded from the analysis. The subjective perception of photopic phenomenon was assessed using the Kruskal-Wallis test; where significance was found, multiple Mann-Whitney tests were performed with a Bonferroni correction (with significance adjusted to $\mathrm{p}<0.017)$. C-Quant and Aston halometer measures were found to be normally distributed (KolmogrovSmirnov test $>0.05$ ) so the resultant data were analysed using repeated measure analysis of variance. Where significance was identified, a Bonferroni post hoc test was used to detail the individual differences between the IOLs. Intraobserver variability of the C-Quant and Aston halometer measurements was determined using intraclass correlation coefficients (ICC). As the eyes of an individual are generally similar, when using monocular data only, the right eye was included in the analysis to avoid statistical bias.

The subjective rating of dysphotopsia was correlated with both measurement of straylight and measurement from the Aston halometer using the Spearman's rank correlation. Correlation between the straylight scores and the area Aston halometer scores was determined using Pearson's correlations.

\section{RESULTS}

Only data from 15 patients with each IOL, achieving a distance visual acuity of $<0.1 \log$ MAR, having centred IOLs (observed through slit-lamp biomicroscopy and optical coherence tomography), no significant posterior subcapsular opacification or had yttrium aluminium garnet (YAG) laser capsulotomy and a 
residual refractive error of $\leq \pm 0.25 \mathrm{D}$ sphere and $\leq-0.50 \mathrm{D}$ astigmatism by subjective refraction, was included in the analysis (14 men, 31 women; mean age $61.8 \pm 8.9$ years). The patient demographics were $60.7 \pm 11.0$ years, $4: 11$ (men:women), in the bilateral Tecnis ZM900 group, $62.3 \pm 9.0$ years, $7: 8$, in the bilateral Lentis Mplus MF30 group and 62.1 \pm 6.8 years, $3: 12$ in the bilateral Softec-1 group. The ages were similar for each IOL group $\left(\mathrm{F}_{2}=0.177, \mathrm{p}=0.838\right)$, hence no age correction was applied.

A significant difference in subjectively rated glare was found between the three groups (H2=12.359, $\mathrm{p}=0.002)$. No significant difference was found between the Softec-1 monofocal group and the Tecnis ZM900 multifocal group $(Z=0.290)$ or between the Softec- 1 monofocal and Lentis Mplus MF30 multifocal groups $(\mathrm{Z}=0.187)$. However, there was a significant difference between the Tecnis ZM900 and the Lentis Mplus MF30 multifocal group $(Z<0.001$; figure 2$)$. No patient reported a difference in glare symptoms between their eyes. Dysphotopsia categorisation is summarised in figure 3 for each of the IOL groups.

There was no significant difference in straylight results between the right and left eyes for each of the IOL groups (Tecnis ZM900, $\mathrm{p}=0.430$; Lentis Mplus MF30, $p=0.513$; Softec-1, $\mathrm{p}=0.902)$. The level of straylight present in the right eyes of each group is displayed in figure 4; all IOL groups demonstrated a similar amount of straylight $\left(\mathrm{f}_{2}=0.414, \mathrm{p}=0.664\right)$. Intraobserver variability for the C-Quant was good $(\mathrm{ICC}=0.765)$.

There was a significant difference in the size of halos when comparing the photopic phenomena in the right eyes $\quad\left(\mathrm{F}_{2,42}=11.288 \quad \mathrm{p}<0.001\right)$ and binocularly $\left(\mathrm{F}_{2,42}=19.525, \mathrm{p}<0.001\right)$. Differences between groups can be seen in figure 5 .

Monocularly and binocularly, the differences were between Tecnis ZM900 and the monofocal control at all eccentricities $(p<0.01)$ and the Lentis Mplus MF30

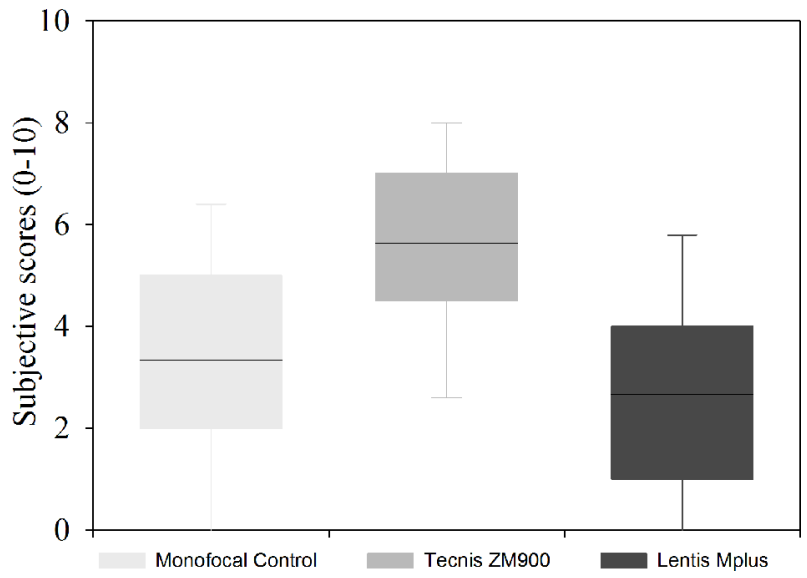

Figure 2 Box plots of subjective 0-10 dysphotopsia scores $(n=15 \times 3)$. Line within box is the median value, box marks extent of 1 standard deviation and error bars indicate the 95\% confidence interval.

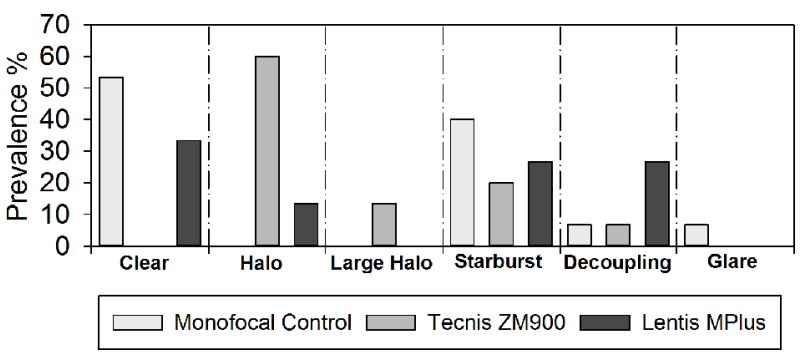

Figure 3 Prevalence of the types of dysphotopsia $(n=15 \times 3)$.

at all eccentricities except $225^{\circ}, 270^{\circ}, 315^{\circ}$ ( $\left.\mathrm{p}>0.05\right)$, whereas the Lentis Mplus MF30 was similar to the monofocal at $180^{\circ}$ (binocularly only) $225^{\circ}$ and $270^{\circ}$ eccentricities $(p>0.05)$. Examination of the monocular $\left(\mathrm{F}_{2}=8.163, \mathrm{p}=0.001\right)$ and binocular $\left(\mathrm{F}_{2}=14.453\right.$, $\mathrm{p}<0.001)$ glare areas revealed a significant difference between the groups. The area of glare was significantly greater in the Tecnis ZM900 group than the Lentis Mplus MF30 $(\mathrm{p}<0.05)$ and monofocal $(\mathrm{p}<0.01)$ group. Glare areas for the Lentis Mplus MF30 and monofocal groups were similar $(p>0.05)$. The intraobserver variability (ICC) of the Aston halometer for each meridian varied from 0.840 to 0.916 (average 0.89 ).

There was no significant correlation between the subjective scores and the straylight scores $\left(r_{s}=-0.103\right.$, $\mathrm{p}=0.503$ ). Similarly no significant correlation was found between the subjective scores and the monocular $\left(r_{\mathrm{s}}=0.246, \quad \mathrm{p}=0.103\right)$ and binocular $\left(\mathrm{r}_{\mathrm{s}}=0.241\right.$, $\mathrm{p}=0.111)$ halometry scores. There was also no significant correlation found between the straylight scores and the halometry area both monocular $(r=0.051$, $\mathrm{p}=0.739)$ and binocular $(\mathrm{r}=0.153, \mathrm{p}=0.315)$.

\section{DISCUSSION}

Except for a recent study, ${ }^{23}$ the use of halometry in studies examining multifocal IOLs has been limited to gross estimation halometry, perimetry halometry, subjective illustration halometry, and the Glare \& Halo test. ${ }^{27}$ All of these halometers (with the exception of perimetry halometry) require the subject to indicate subjectively the boundaries of their photopic scotoma. Of the studies that have used a halometer to examine

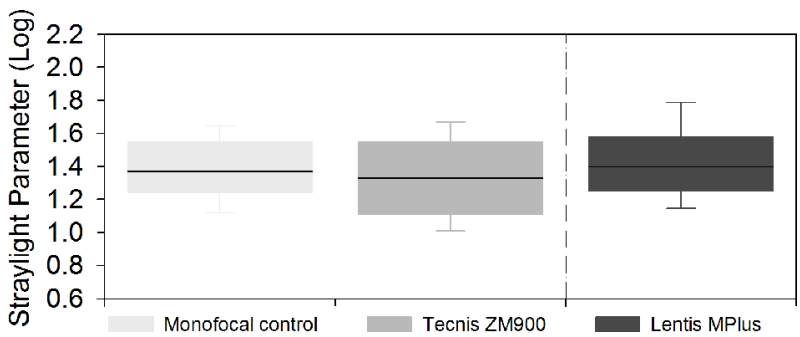

Figure 4 Level of straylight for each IOL group $(n=15 \times 3)$. Line within box is the median value, box marks extent of 1 standard deviation and error bars indicate the 95\% confidence interval. 

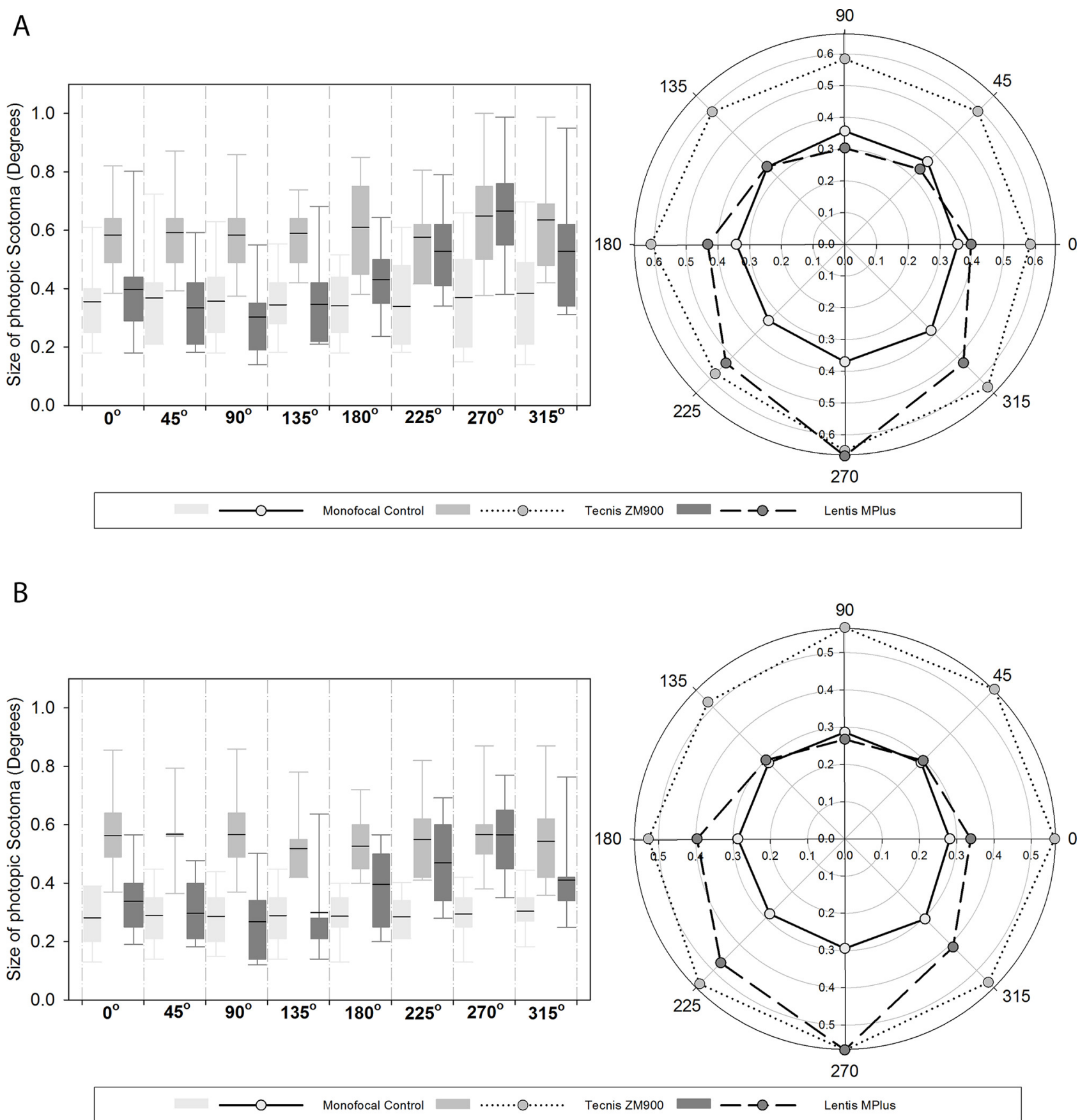

Figure 5 Monocular (top) and binocular (bottom) results of the Aston Halometer for each of the IOL groups. Right polar plot, left box plots $(n=15 \times 3)$. Line within box is the median value, box marks extent of 1 standard deviation and error bars indicate the $95 \%$ confidence interval.

dysphotopsia with multifocal IOL subjects, five failed to demonstrate an increase in dysphotopsia after multifocal IOL implantation. 92329 However, a common complaint after multifocal IOL implantation is an increase in dysphotopsia. ${ }^{12}$

This study shows that the Aston Halometer is a repeatable and sensitive method for the assessment of dysphotopsia. The C-Quant detected no significant differences in the level of straylight regardless of the implanted IOL; this concurs with some, ${ }^{2}{ }^{17-19}$ but not all $^{14-16}$ previous studies and suggests that measures of straylight are not inferred measures of dysphotopsia caused by multifocal IOLs. Of note, the C-Quant values were on the higher side of what has been reported as the average for this age group, ${ }^{19}$ but this was the case with all three IOL groups.

At each meridian the Tecnis ZM900 multifocal IOL displayed a larger amount of photopic scotoma in comparison with the Softec-1 monofocal IOL. This is in keeping with the known descriptions of haloes caused by multifocal IOLs ${ }^{12}$ and predominance of 'halo' described with the EyeVisPod. The Lentis Mplus MF30-segmented multifocal IOL did not demonstrate the same appearance; superiorly, the photopic scotoma region was 
similar to the monofocal IOL; however, in the inferior portion of the visual field (around $270^{\circ}$ ), there was a greater amount of scotoma similar to the levels exhibited by the Tecnis ZM900 multifocal IOL; this can be explained by the implanted location of the reading portion of the lens. In all subjects, the Lentis Mplus MF30 IOL was implanted with the reading portion inferiorly, resulting in the defocused rays from the second focal point falling on the superior portion of the retina, responsible for the inferior visual field. As well as in the $270^{\circ}$ position, the levels of photopic scotoma with the Lentis Mplus MF30 was greater in the $180^{\circ}$ and $225^{\circ}$ positions binocularly and in the $225^{\circ}$ position monocularly. This may suggest that not all of the lenses were placed accurately with the reading segment in the inferior portion of the lens or rotated from this position and may explain an increased SD in the inferior regions.

The subjective categorisation of dysphotopsia supports the halometry results; approximately $73 \%$ of the patients implanted with Tecnis ZM900 multifocal IOL associated their dysphotopsia with either the small or large halo images, whereas only $13 \%$ of the Lentis Mplus MF30 segmented multifocal IOL group associated their vision with the halo illustration, with 53\% of this group reporting either the starburst or decoupling image. The decoupling image is of interest as it depicts a ghost image inferiorly to the light sources on screen. Subjective dysphotopsia ratings were able to differentiate the Tecnis ZM900 from the Lentis Mplus MF30 multifocal IOLs, but not the Lentis Mplus MF30segmented multifocal IOL from the group implanted with Softec-1 monofocal IOL. There was no correlation between the overall subjective scores and the straylight and halometry results. These findings highlight the issue with global ratings of temporally and spatially variable symptoms, probably confounded by the variability found with subjective rating scales and hence support the need for an objective method of testing for quantifying haloes and glare. Adaption and tolerance to dysphotopsia are additional factors that need to be considered and may further explain the disparity between subjective and objective measures. For example, type 'A' personality traits are regarded as a risk factor for multifocal IOL implantation as this group is widely regarded as intolerant to dysphotopsia. ${ }^{30}$

Standardised halometry may be useful in determining other risk factors for multifocal rejection. Considering the efficacy of halometry and the potential of multifocal IOLs to exacerbate symptoms of glare, a standardised preoperative measure of halo size compared with subjectively reported symptoms due to cataract, might help to identify individuals who are more likely to better tolerate dysphotopsia effects potentially induced by multifocal IOLs. For example, the larger the tolerance ratio of objective (halometry measure halo size) divided by subjective (self-reported) glare before cataract surgery, the less likely a patient should be to complain about glare symptoms with an implanted multifocal IOL after surgery.

The study did not randomise patients between the IOLs, but each surgeon implanted a similar number of each design, the patients had a similar age profile and the comparison between methods to assess dysphotopsia were assessed across all patients, so the findings of the study should not have been compromised by this methodology.

In conclusion, the Aston halometer is a repeatable and sensitive method able to differentiate between multifocal IOL dysphotopsia, unlike retinal straylight assessment of veiling luminance over the whole retina. $^{28}$ The fully diffractive multifocal IOL demonstrated a uniform increase in dysphotopsia in comparison with the monofocal IOL as measured with halometry, whereas the sectorial refractive multifocal IOL demonstrates a localised increase in dysphotopsia over the inferior visual field.

Acknowledgements Mark T Benson and Tina A Kipioti implanted some of the IOLs. T

Contributors Authors were involved in the design (PJB, SAN, LND, SS, JSW), data collection (PJB, SS, TD), analysis (PJB, JSW) and manuscript writing/ approval (PJB, SAN, LND, SS, TD, JSW) for this study. This study forms part of PB's doctoral thesis.

Competing interests None declared.

Ethics approval NHS local research ethics committee of Solihull.

Provenance and peer review Commissioned; externally peer reviewed.

Data sharing statement The authors are willing to receive requests to share the data collected in this study, all of which is included in this manuscript.

Open Access This is an Open Access article distributed in accordance with the Creative Commons Attribution Non Commercial (CC BY-NC 4.0) license, which permits others to distribute, remix, adapt, build upon this work noncommercially, and license their derivative works on different terms, provided the original work is properly cited and the use is non-commercial. See: http:// creativecommons.org/licenses/by-nc/4.0/

(c) Article author(s) (or their employer(s) unless otherwise stated in the text of the article) 2017. All rights reserved. No commercial use is permitted unless otherwise expressly granted.

\section{REFERENCES}

1. Leyland M, Zinicola E. Multifocal versus monofocal intraocular lenses in cataract surgery: a systematic review. Ophthalmology 2003;110:1789-98.

2. Wilkins MR, Allan BD, Rubin GS, et al. Randomized trial of multifocal intraocular lenses versus monovision after bilateral cataract surgery. Ophthalmology 2013;120:2449-55.

3. Jacobi PC, Dietlein TS, Lueke C, et al. Multifocal intraocular lens implantation in patients with traumatic cataract. Ophthalmology 2003;110:531-8.

4. Marques EF, Ferreira TB. Comparison of visual outcomes of 2 diffractive trifocal intraocular lenses. J Cataract Refract Surg 2015;41:354-63.

5. Kohnen T, Allen D, Boureau C, et al. European multicenter study of the AcrySof ReSTOR apodized diffractive intraocular Lens. Ophthalmology 2006;113:578-84.

6. Aslam TM, Dhillon B, Tallentire VR, et al. Development of a forced choice photographic questionnaire for photic phenomena and its testing - repeatability, reliability and validity. Ophthalmologica 2004;218:402-10.

7. Aslam TM, Gilmour D, Hopkinson S, et al. The development and assessment of a self-perceived quality of vision questionnaire to test pseudophakic patients. Ophthalmic Epidemiol 2004;11:241-53. 
8. Shoji N, Shimizu K. Clinical evaluation of a $5.5 \mathrm{~mm}$ three-zone refractive multifocal intraocular lens. J Cataract Refract Surg 1996;22:1097-101.

9. Hunkeler JD, Coffman TM, Paugh J, et al. Characterization of visual phenomena with the Array multifocal intraocular Lens. J Cataract Refract Surg 2002;28:1195-204.

10. McAlinden C, Pesudovs K, Moore JE. The development of an instrument to measure quality of vision: the Quality of Vision (QoV) questionnaire. Invest Ophthalmo/ Vis Sci 2010;51:5537-45.

11. Vos JJ. Disability glare state of the art report. CIE-Journal 1984;3:39-53.

12. Bailey IL, Bullimore MA. A new test for the evaluation of disability glare. Optom Vis Sci 1991;68:911-7.

13. Epitropoulos AT, Fram NR, Masket S, et al. Evaluation of a new controlled point source LED glare tester for disability glare detection in participants with and without cataracts. J Refract Surg 2015;31:196-201.

14. de Vries NE, Franssen $L$, Webers CA, et al. Intraocular straylight after implantation of the multifocal AcrySof ReSTOR SA60D3 diffractive intraocular lens. J Cataract Refract Surg 2008;34:957-62.

15. Peng $\mathrm{C}$, Zhao J, Ma L, et al. Optical performance after bilateral implantation of apodized aspheric diffractive multifocal intraocular lenses with +3.00-D addition power. Acta Ophthalmol 2012;90:e586-e593.

16. Lapid-Gortzak R, Labuz G, van der Meulen IJ, et al. Straylight measurements in two different apodized diffractive multifocal intraocular lenses. J Refract Surg 2015;31:746-51.

17. Hofmann T, Zuberbuhler B, Cervino A, et al. Retinal straylight and complaint scores 18 months after implantation of the AcrySof monofocal and ReSTOR diffractive intraocular lenses. J Refract Surg 2009;25:485-92.

18. Cerviño A, Hosking SL, Montés-Micó R, et al. Retinal straylight in patients with monofocal and multifocal intraocular lenses. $J$ Cataract Refract Surg 2008;34:441-6.

19. Łabuz G, Reus NJ, van den Berg TJ, et al. Comparison of ocular straylight after implantation of multifocal intraocular lenses. $J$ Cataract Refract Surg 2016;42:618-25.
20. Ehmer A, Rabsilber TM, Mannsfeld A, et al. Influence of different multifocal intraocular lens concepts on retinal stray light parameters. Ophthalmologe 2011;108:952-6.

21. Ferreira-Neves $\mathrm{H}$, Macedo-de-Araújo R, Rico-Del-Viejo L, et al. Validation of a method to measure light distortion surrounding a source of glare. J Biomed Opt 2015;20:075002.

22. Gutiérrez R, Jiménez JR, Villa $C$, et al. Simple device for quantifying the influence of halos after lasik surgery. J Biomed Opt 2003;8:663-7.

23. Brito $\mathrm{P}$, Salgado-Borges J, Neves $\mathrm{H}$, et al. Light-distortion analysis as a possible indicator of visual quality after refractive lens exchange with diffractive multifocal intraocular lenses. J Cataract Refract Surg 2015;41:613-22.

24. Guo YW, Li J, Song H, et al. Comparison of the retinal straylight in pseudophakic eyes with PMMA, hydrophobic acrylic, and hydrophilic acrylic spherical intraocular lens. J Ophthalmol 2014;2014:1-6.

25. Babizhayev MA, Minasyan H, Richer SP. Cataract halos: a driving hazard in aging populations. implication of the Halometer DG test for assessment of intraocular light scatter. Appl Ergon 2009;40:545-53.

26. Meikies D, van der Mooren M, Terwee T, et al. Rostock glare perimeter: a distinctive method for quantification of glare. Optom Vis Sci 2013;90:1143-8

27. Buckhurst PJ, Naroo SA, Davies LN, et al. Tablet app halometer for the assessment of dysphotopsia. J Cataract Refract Surg 2015;41:2424-9.

28. Guber I, Bachmann LM, Guber J, et al. Reproducibility of straylight measurement by C-Quant for assessment of retinal straylight using the compensation comparison method. Graefes Arch Clin Exp Ophthalmol 2011;249:1367-71.

29. Łabuz G, Vargas-Martín F, van den Berg TJ, et al. Method for in vitro assessment of straylight from intraocular lenses. Biomed Opt Express 2015;6:4457-64.

30. Koch DD, Wang L. Custom optimization of intraocular Lens asphericity. Trans Am Ophthalmol Soc 2007;105:36-41. 